\title{
Propagation of travelling waves in a complex system modelling fire spread
}

\author{
$\underline{\text { S. Watt }}^{\mathrm{a}}$, H. Sidhu ${ }^{\mathrm{a}}$, Z. Jovanoski ${ }^{\mathrm{a}}$, I. Towers ${ }^{\mathrm{a}}$, J. Sharples ${ }^{\mathrm{a}}$ and V. Gubernov ${ }^{\mathrm{b}}$

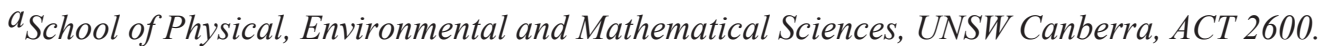 \\ b. E. Tamm Theory Department, P.N. Lebedev Physical Institute, 53 Leninsky Prospect, 119991 Moscow, \\ Russian Federation \\ Email: simon.watt@adfa.edu.au
}

\begin{abstract}
A system of coupled partial differential equations which models a complex system of a solid fuel, endothermically pyrolysing to a combustible gas, which in turn exothermically reacts with oxygen, is studied. We use a numerical method based on the Crank-Nicholson finite difference scheme to solve the governing equations in order to investigate the dynamics of this model. It has previously been shown that there exist solutions to the model which exhibit oscillatory propagating combustion waves. In this work, we extend the original study to explore the parameter space to locate regions where both steady propagating waves (single valued wave speed) and also pulsating waves exist. Parameter space where no propagating waves are possible (extinction region) is also determined.
\end{abstract}

Keywords: Combustion, exothermic, endothermic, pyrolysis, travelling waves, instability, bifurcation 


\section{INTRODUCTION}

The modelling of fire spread has been an active research topic with many applications to both urban and rural fire situations, particularly to minimise risk to humans, livestock and infrastructure (Drysale 1999, Rahmawati et al. 2016, Peng et al. 2016). Of particular interest is the dynamics of fire spread (Grishin 1984, Weber 1991, Duane et al. 2016). The rate of spread of the combustion wave is of interest, for example in the case of wildfire spread (Watt et al. 1995, Hilton et al. 2015).

In previous work by Weber et al. (2001), a model was presented which involved the combustion of a solid fuel which underwent an endothermic gasification which then could react exothermically with oxygen, and with the heat released to propagate a combustion wave. One of the results of the work was the discovery of oscillatory propagating combustion waves for a given set of system parameters. In the present investigation, we revisit this model with the intention to determine various dynamical behavior in the parameter space.

In studying travelling wave solutions in combustion problems (see for example Volpert et al. (1994)), the existence of a stable, steady propagating combustion wave is assumed. For some systems, it is possible to obtain an asymptotic approximation to the wave speed (Weber et al. 1997). However, as with dynamical systems, it has been shown that in a system of partial differential equations (PDEs) there is the possibility of instabilities (Gubernov et al. 2003, 2004). The existence of possible bifurcation of wave speed was first noticed by Shkadinskii et al. (1971). In Weber et al. (1997), a single-step combustion model was demonstrated to have an instability as the system parameters were varied and a period-doubling route to chaos was shown. Similar analysis was carried out for more complicated models such as for a competitive exothermic-endothermic combustion problem (Sharples et al. 2012, Wee et al. 2012) and for a sequential reaction (Qian et al. 2011).

\section{MATHEMATICAL MODEL}

We begin with the conservation of energy and mass, for the solid and gas phases. This model is based on that presented in Weber et al. (2001) where it was assumed that the solid fuel cannot diffuse heat or mass, while the gaseous fuel can diffuse. The non-dimensionalised system can be written as

$$
\begin{aligned}
\frac{\partial u_{s}}{\partial t} & =-Q_{s} v_{s} e^{-\gamma / u_{s}}+h_{s}\left(u_{g}-u_{s}\right)-\ell_{s}\left(u_{s}-u_{a}\right), \\
\frac{\partial v_{s}}{\partial t} & =-\beta_{s} v_{s} e^{-\gamma / u_{s}}, \\
\frac{\partial u_{g}}{\partial t} & =\frac{\partial^{2} u_{g}}{\partial x^{2}}+Q_{g} v_{g} e^{-1 / u_{g}}-h_{g}\left(u_{g}-u_{s}\right)-\ell_{g}\left(u_{g}-u_{a}\right), \\
\frac{\partial v_{g}}{\partial t} & =\frac{1}{L e} \frac{\partial^{2} v_{g}}{\partial x^{2}}-\beta_{g} v_{g} e^{-1 / u_{g}}+\beta_{s} v_{s} e^{-\gamma / u_{s}},
\end{aligned}
$$

where $u$ denotes temperature and $v$ denotes fuel density, the subscripts $g$ and $s$ correspond to the gaseous and solid stages respectively, $Q$ denotes heat of combustion, $h$ is a heat transfer between the phases, $\ell$ is a heat loss coefficient, $u_{a}$ is the ambient temperature and $\beta$ are the stoiciometric coefficients of the phases. In addition there are two relative parameters, $\gamma$ which is a ratio of the activation energies of the two reactions and Le is the Lewis number and is the ratio of the thermal conductivity and the mass diffusivity in the gaseous phase.

\section{NUMERICAL METHOD}

The system (1) - (4) was solved numerically for different stoiciometric coefficients $\left(\beta_{s}\right.$ and $\left.\beta_{g}\right)$ using the Crank-Nicholson method (see for example Press et al. (1992)) with a finite difference scheme for the spatial derivatives and adaptive finite difference for the time derivatives. The Crank-Nicholson method was chosen as it is unconditionally stable for any time step. However the time step is adjusted to satisfy error tolerances.

For each of the numerical solutions, the initial temperature and fuel profiles are

$$
u_{s}=\left[1-H\left(x-x^{*}\right)\right] u^{*}+u_{a}, \quad v_{s}=1, \quad u_{g}=u_{a} \text { and } v_{g}=0,
$$

where $H$ is the Heaviside step-function, $u^{*}$ and $x^{*}$ are the height and width of the step function respectively. We also impose the following boundary conditions

$$
\begin{array}{r}
\frac{\partial u_{s}}{\partial x}=\frac{\partial v_{s}}{\partial x}=\frac{\partial u_{g}}{\partial x}=\frac{\partial v_{g}}{\partial x}=0 \text { at } x=0, \\
u_{s}=u_{a} \quad v_{s}=1 \quad u_{g}=u_{a} \quad v_{g}=0 \text { at } x=L,
\end{array}
$$




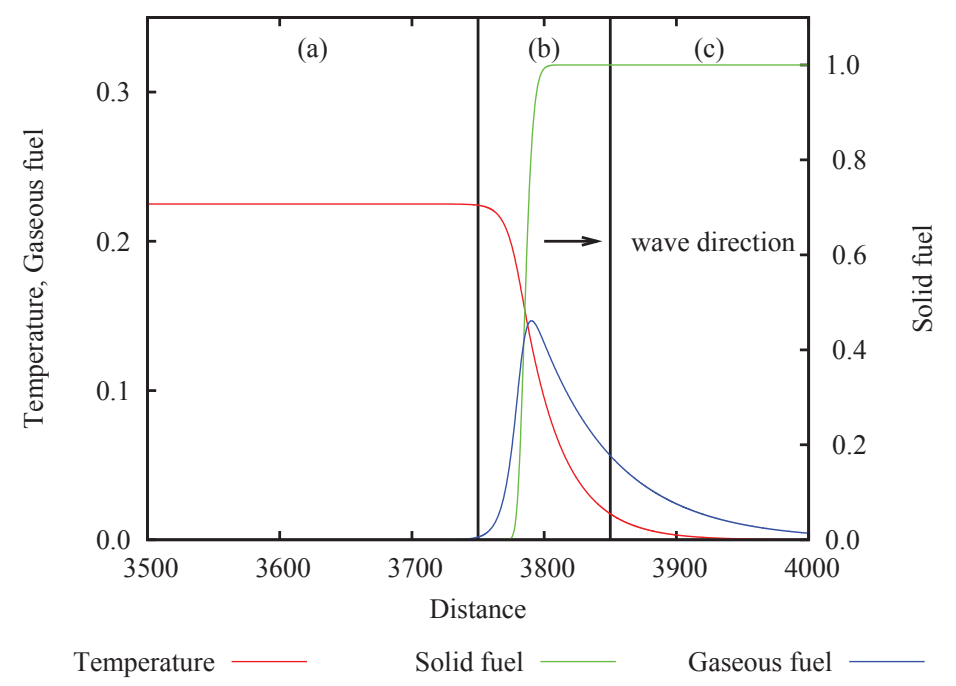

Figure 1. A schematic of the three characteristic zones in the travelling wave solution (a) the "hot" burnt zone (b) the reaction zone and (c) the "cold" unburnt zone.

where $x=0$ and $x=L$ are the left and right boundaries respectively.

The system was then numerically integrated for a sufficiently long period so that the solution renders a steadystate solution. The progress was monitored to ensure that spatial domain was large enough to ignore boundary effects. The size of the step-function was adjusted such that there was sufficient initially energy in the system to sustain a possible combustion wave. The numerical solutions obtained by the Crank-Nicholson method were independently validated using a commericial finite element package FlexPDE (PDE Solutions Inc. 2010). The solutions obtained using both of these approaches were found to be within $1 \%$ of each other.

\section{NUMERICAL RESULTS}

In this work, we solve the system (1) - (4) numercially using the parameters provided in Weber et al. (2001) which were $L e=1, Q_{s}=0.1, Q_{g}=1.0, h_{s}=h_{g}=0.1, \gamma=1.0$ and $l_{s}=l_{g}=0$. This last condition means we are considering the adiabatic case when there is no heat loss. The two stoiciometric parameters $\beta_{g}$ and $\beta_{s}$ will be varied to obtain the different behavior of the travelling wave solution. In the solutions, the travelling wave is assumed to progress from the left to the right of the domain. A typical steady state solution of a combustion wave travelling with constant speed is shown in Figure 1.

There are three main regions (a) behind the travelling wave where the solid fuel has been used, pyrolysis has ceased and the temperature is in a "hot" state (b) at the wave front where the solid fuel is being pyrolysed through combustion and (c) ahead of the travelling wave where the solid fuel is unburnt and no pyrolysis has occurred. In the figure, there is also diffusion of the temperature and gaseous fuel ahead of the reaction zone.

It should be noted that as there is a coupling term between the solid and gaseous temperatures, the two temperatures quickly evolve to the same system temperature. By using this assumption, it is possible to take a linear combination of the system equations (1) - (4) to derive the following result for the temperature of the "hot" state at the left boundary

$$
u=u_{g}=u_{s}=\frac{h_{s} h_{g}}{h_{s}+h_{g}}\left(\frac{Q_{g}}{h_{g} \beta_{g}}-\frac{Q_{s}}{h_{s} \beta_{s}}\right) .
$$

\subsection{Steady wave speed}

For the stoiciometric parameter $\beta_{g}=\beta_{s}=2$, we have found solutions which evolve to a steady wave speed, as shown in Figure 2. In this (and subsequent figures), the combustion wave travels from left to right. As the system is spatially invariant, there would exist a combustion wave travelling in the opposite direction for $x<0$. From the figure, the solid and gaseous fuel profiles are a constant shape over time. The temperature 

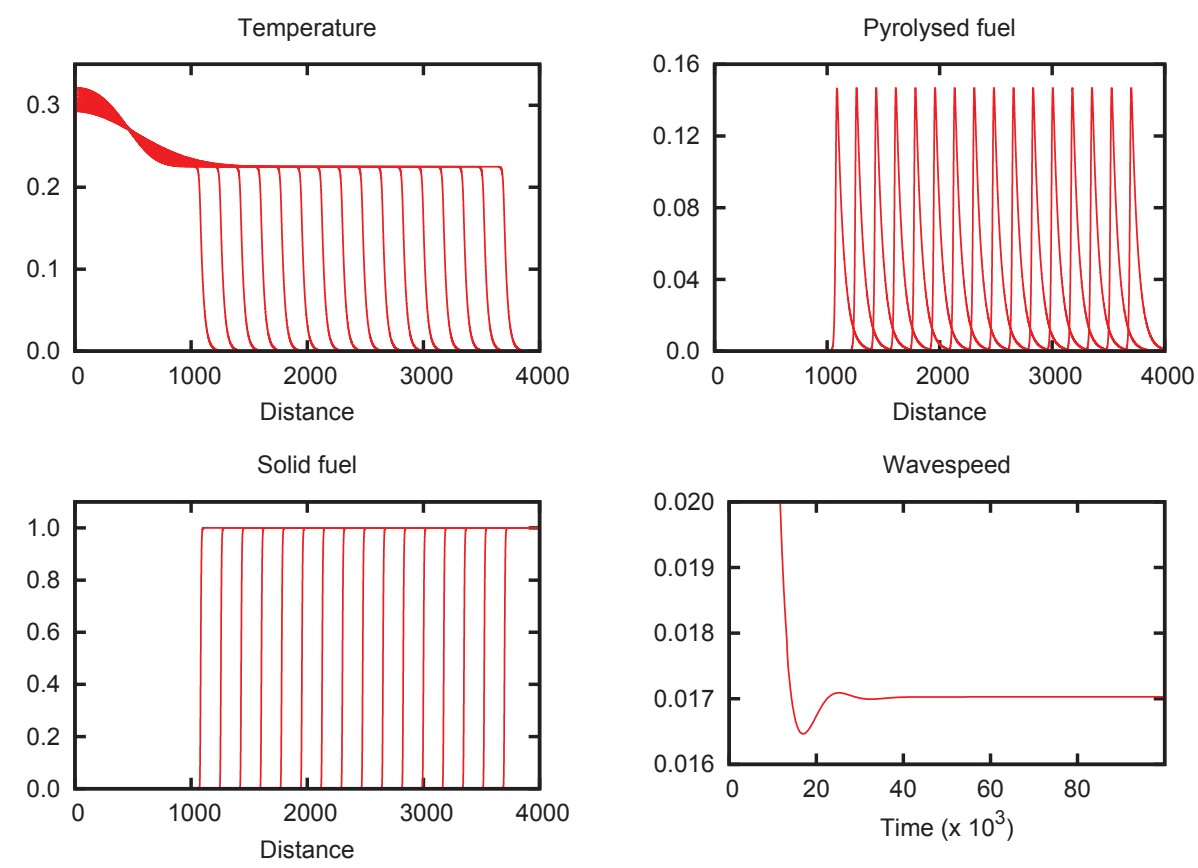

Figure 2. Travelling wave solution with steady wave speed $(c=0.017)$ with $\beta_{g}=\beta_{s}=2$, and all parameter values given in the text.

profile is mostly a constant shape over time except on the left hand side (around $0<x<1000$ ). In this region, the wave front has passed and there is very little solid or gaseous fuel to drive the reaction. So effectively in this region, the evolution of the temperature profile $(u)$ is governed by

$$
\frac{\partial u}{\partial t}=\frac{\partial^{2} u}{\partial x^{2}}
$$

This has a fundamental solution (see for example Watt et al. (1995))

$$
u=\frac{A}{\sqrt{2 \pi(t+B)}} e^{-x^{2} / 4\left(t+t_{1}\right)}+C,
$$

where $A$ and $B$ are fitted parameters and $C=u(0, t)$ is calculated from (5). So the temperature profile behind the wave front will decay at the reciprocal of the square root of time.

\subsection{Oscillatory wave speed - pulsating waves}

As the stoiciometric parameters are increased, as seen in the single-step model (Weber et al. 1997) and the results reported in Weber et al. (2001), an instability develops in the wave speed solition and a periodic solution for the wave speed is found for $\beta_{g}=\beta_{s}=3$. The solution is shown in Figure 3. The solution looks fundamentally different when compared to the constant wave speed case (c.f. Figure 2). The main difference is that the profiles of the temperature and pyrolysed fuel are no longer a constant shape. In particular, the peak of the wave profiles for the temperature and pyrolsed fuel are oscillating. The other notable difference is the banding or surging of the solid fuel profile. This indicates pulsating behavior when the wave speed accelerates and decelerates in a periodic manner. We will refer to this solution as period-1 given the wave speed profile.

Increasing the stoiciometric parameters further, as has been found in similar work (for example Qian et al. (2011)), to $\beta_{g}=\beta_{s}=3.3$, the period-1 solution becomes unstable and the solution evolves to one with a period-2 wave speed (shown in Figure 4). The period-1 and period-2 solutions shown in Figures 3 and 4 are similar in that they both show banding in the solid fuel and the oscillation of the peaks of the temperature and gaseous profiles. One of the significant differences are the different spatial and time scales between the two. Comparing the period- 2 to the period- 1 solutions, the numerical integration that was required to run to obtain 

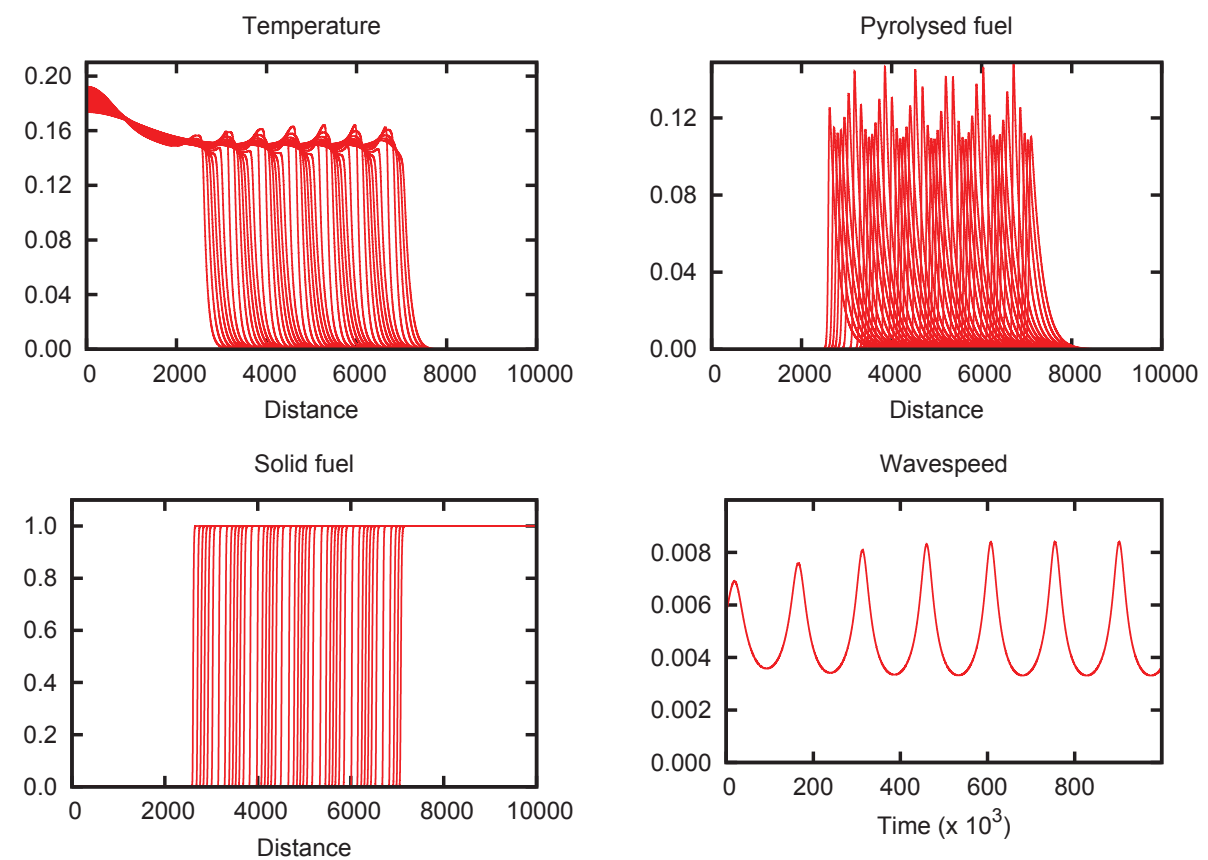

Figure 3. Travelling wave solution with oscillatory wave speed with $\beta_{g}=\beta_{s}=3$ - period-1 solutions.
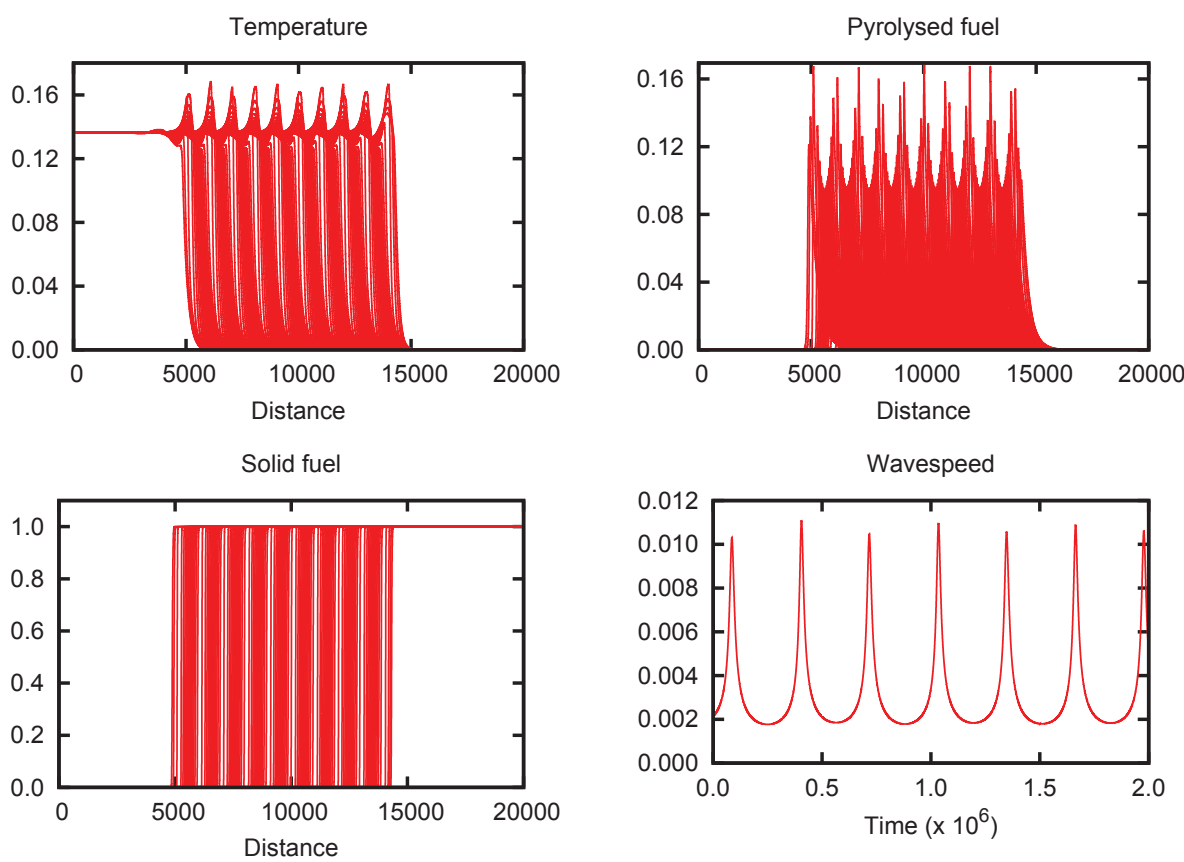

Figure 4. Travelling wave solution with oscillatory wave speed for $\beta_{g}=\beta_{s}=3.3$ - period-2 solutions.

a steady-state solution for the former case is over a longer spatial domain and time period when compared to the period-1 solution. The average wave speed for the period- 2 solution was around ten times slower.

\section{EXPLORING THE PARAMETER SPACE}

To get a better understanding of the system and to follow similar analysis such as that carried out in Qian et al. (2011), we explored the $\beta_{s}-\beta_{g}$ parameter space and classified the travelling wave solutions as either (a) steady wave speed (b) oscillatory wave speed (this could also include higher periodic solutions rather than the 
period-1 and period-2 solutions shown in the previous section) and (c) extinction waves where no propagating combustion waves persist. For a given set of parameter values, the system (1) - (4) were numerically integrated until one of the three solution types were found.

The different types of solutions for various values of the stoiciometric parameters is shown in Figure 5. There is a Hopf curve which separates the parameter space between solutions that exhibit steady (constant speed) waves and oscillatory (pulsating) waves. This Hopf curve marks the onset of periodic behavior and was found through a manual path-following method through the paramater space. The Extinction curve describes the locus of points in the parameter space where the "hot" temperature condition found in Equation (5) was zero. In this case, the temperature diffuses away once the solid and gaseous fuels have been consumed and no combustion wave can exist. The point where the Hopf curve and the Extinction curve intersect (labelled $B$ at $\beta_{s}=0.113, \beta_{g}=1.13$ ) is a possible Bogdanov-Takens bifurcation point, which is a critical equilibrium point (singularity) where saddle-node and Hopf bifurcations occur simultaneously. This has been observed and studied in the context of combustion waves by Gubernov et al. (2004). Whether this is in fact a BogdanovTakens bifurcation point will be investigated in future work.

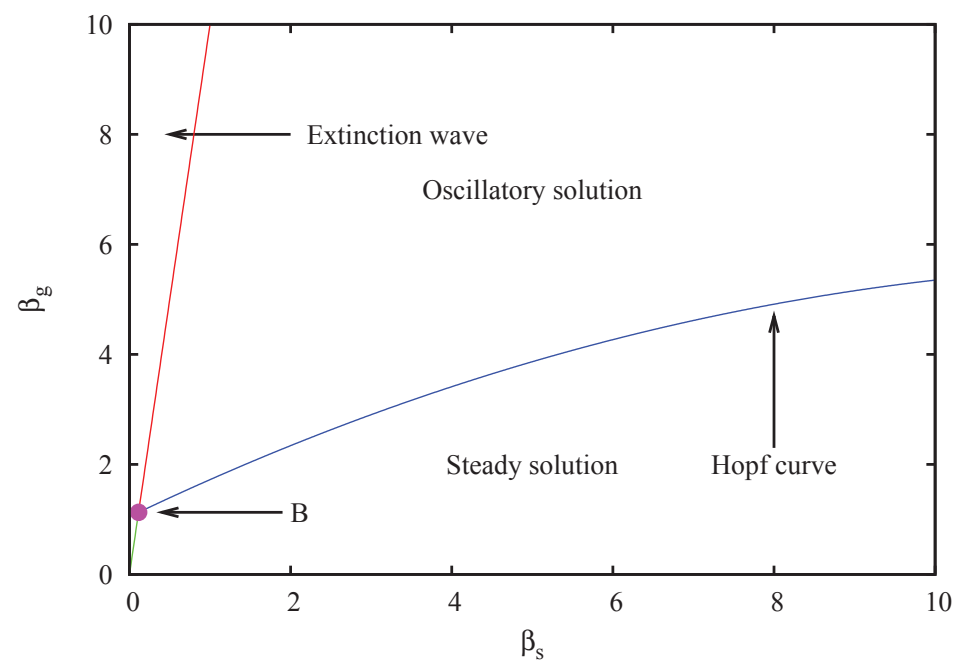

Figure 5. The $\beta_{s}-\beta_{g}$ parameter space showing the different type of waves solutions: steady speed, oscillatory speed and extinction.

\section{CONCLUSIONS}

In this paper, we have undertaken a preliminary investigation of a model describing the combustion scheme with two different phases, namely a solid phase and a gaseous phase. In particular, we were interested in the travelling wave behavior for different physical and chemical parameters. The model that was considered was presented in Weber et al. (2001). In this paper, we extend the original work by investigating the parameter space to locate regions where different dynamical behavior occur - steady combustion waves with a unique wave speed, pulsating waves (oscillatory wave speed) and no combustion waves. In our future work, we plan to undertake a detailed analysis of this coupled system by studying the stability of the combustion waves via the Evans function approach (see for example Gubernov et al. $(2003,2004)$ ).

Finally, it is also important to note that using the parameter values given in Weber et al. (2001), we were not able to replicate the results presented in their paper, but instead discovered "slow" travelling wave solutions (in other words, the wave speeds were much lower than those reported in the original paper). As noted before, we have validated the numercial schemes used extensively in this work by using both the Crank-Nicholson finite difference method and the finite element package FlexPDE (PDE Solutions Inc. 2010). Hence we are confident the numerical results presented in this paper are both accurate and reliable. We intend to explore the differences between our results and those of Weber et al. (2001) further to ascertain whether there were errors in the published values given in the original paper, or there may well indeed be two families of solutions - the 
"fast" and "slow" travelling wave solutions for the same parameter values. This bistability in travelling wave solutions where both "fast" and "slow" solution branches co-exist were found in the competitive exotherminicreaction scheme by Huang et al. (2016). Such a case may also exist for the system studied in this paper which could explain the differences between the results.

\section{REFERENCES}

Drysale, D. (1999), An introduction to fire dynamics, John Wiley and Sons, Ltd, Chichester, UK.

Duane, A., Aquilué, N., Gil-Tena, A. \& Brotons, L. (2016), 'Integrating fire spread patterns in fire modelling at landscape scale’, Environmental Modelling and Software 86, 219-231.

Grishin, A. (1984), 'Steady-state propagation of the front of a high-level forest fire', Sov. Phys. Dokl. 29, 917919.

Gubernov, V., Mercer, G., Sidhu, H. \& Weber, R. (2003), 'Evans function stability of combustion waves', SIAM Journal on Applied Mathematics 63(4), 1259-1275.

Gubernov, V. V., Mercer, G. N., Sidhu, H. S. . \& Weber, R. O. (2004), 'Evans function stability of non-adiabatic combustion waves', Proc. R. Soc. Lond. A 460, 2415-2435.

Hilton, J., Miller, C., Sullivan, A. \& Rucinski, C. (2015), 'Effects of spatial and temporal variation in environmental conditions on simulation of wildfire spread', Environmental Modelling and Software 67, 118-127.

Huang, Z., Sidhu, H. S., Towers, I. N., Jovanoski, Z. \& Gubernov, V. V. (2016), 'Nonadiabatic combustion waves in a two-step competitive exothermic-reaction model', ANZIAM J. 57, C14-C31.

PDE Solutions Inc. (2010), 'Flexpde'.

Peng, K., Chu, L. \& Li, J. (2016), 'Research and countermeasures of fire protection in southwest rural areas', Procedia Engineering 135, 197 - 206.

Press, W., Teukolsky, S., Vetterling, W. \& Flannery, B. (1992), Numerical Recipes in C: The Art of Scientific Computing, Cambridge University Press, New York.

Qian, C., Sidhu, H., Sharples, J., Towers, I. \& Gubernov, V. (2011), Combustion waves from a sequenctial exothermic and endothermic reaction, in 'Proceedings of MODSIM2015', pp. 725-732.

Rahmawati, D., Pamungkas, A., Ulfa Aulia, B., Dyah Larasati, K., Aditya Rahadyan, G. \& Hardwin Dito, A. (2016), 'Participatory mapping for urban fire risk reduction in high-density urban settlement', Procedia Social and Behavioral Sciences 227, 395-401.

Sharples, J., Sidhu, H., McIntosh, A., Brindley, J. \& Gubernov, V. (2012), 'Analysis of combustion waves arising in the presence of a competitive endothermic reaction', IMA Journal of Applied Mathematics 77(1), 1831 .

Shkadinskii, K., Khaikin, B. \& Merzhanov, A. (1971), 'Propagation of a pulsating exothermic reaction front in the condensed phase', Combust. Explosion Shock Waves 7, 15-22.

Volpert, A., Volpert, V. \& Volpert, V. (1994), Travelling wave solutions of parabolic systems, American Mathematical Society, Providence, RI.

Watt, S., Roberts, A. \& Weber, R. (1995), 'Dimensional reduction of a bushfire model', Mathematical and Computer Modelling 21(9), 79-83.

Weber, R. (1991), 'Towards a comprehensive wildfire spread model', Int. J. Wile Fire 1, 245-248.

Weber, R., Mercer, G. \& Sidhu, H. (1997), 'Combustion waves for gases $(L e=1)$ and solid $(L e \rightarrow \infty)$ ', Proc. R. Soc. Lond. A 453, 1105-1118.

Weber, R., Mercer, G. \& Sidhu, H. (2001), Instability of travelling waves in a complex system modelling fire spread, in 'Proceedings of MODSIM 2001', pp. 973-976.

Wee, W., Sidhu, H., Sharples, J., Towers, I. \& Gubernov, V. (2012), 'Properties of reaction fronts in a nonadiabatic two stage exothermic-endothermic competitive reaction scheme', ANZIAM Journal 54, C646C663. 